\title{
High Resolution Multiple Labeling for Immuno-EM applying Metal Colloids and Energy Filtering Transmission Electron Microscopy (EFTEM)
}

\author{
R. Bleher, D.A. Meyer and R.M. Albrecht \\ Department of Animal Sciences, University of Wisconsin, Madison, WI 53706
}

Currently we are developing a methodology for multiple labeling immuno-EM at high resolution with conjugates of metal colloids and primary antibodies. The colloids are made of Au-, Pd- and PtAu spheres approximately $6 \mathrm{~nm}$ in diameter $\left(\mathrm{cAu}_{6}, \mathrm{cPd}_{6} \text { and } \mathrm{cRhAu}\right)_{6}$. After labeling, bound conjugates on ultrathin sections are identified by electron-spectroscopic-imaging (ESI). With this approach, labeling reactions can be compared directly, since labels are identified by their elemental composition. Also, the number of different reporters for multiple labeling immuno-EM is increased in comparison to conventional techniques.

The classical system for multiple labeling immuno-EM uses electron dense markers of different sizes, commonly made of $\mathrm{Au}$, which need to differ considerably in size to prevent overlapping of signals and to allow unambiguous allocation of labels to antigens [1]. Comparison of results obtained with different sized markers is problematic, because larger markers, when bound to an antigen, may block more neighboring binding sites than smaller particles, or they can mask smaller labels thereby making them undetectable. Also, larger particles may bind to more than one epitope per bead, thus making it difficult to compare or quantify signal densities. Additionally, spatial resolution is substantially reduced with larger marker sizes [2].

We used skeletal muscle tissue as a model system for multiple labeling studies because the well known structure of sarcomeres facilitated evaluation of labeling patterns. Anti-alpha-actinin-c $\mathrm{Au}_{6}$ conjugates exclusively labeled Z-lines, whereas anti-actin-c $\mathrm{Pd}_{6}$ and anti-myosin-cPtAu $\mathrm{Au}_{6}$ marker distributions were restricted to I- and A-Bands, or A-Bands only, respectively (Fig. 1, 2).

While $\mathrm{cAu}_{6}$ was generated by the white phosphorous-method [3], for the production of $\mathrm{cPd}_{6}, \mathrm{a}$ substantially modified citrate/tannic acid-method was used [4, 5]. cPtAu ${ }_{6}$ colloids were obtained using an adaptation of the core-shell technique [6].

Metal labels were identified in a LEO 912 EFTEM equipped with an Omega energy filter by ESI [7]. For ESI, inelastically scattered electrons with element specific energy losses can be selectively used for imaging to generate elemental distribution maps. We applied the three windows method, in which two images are taken at energy losses below the element specific energy loss maximum to extrapolate the background signal which is then subtracted from the third image at the maximum element specific energy loss [8].

References

[1] W.P. Faulk and G.M. Taylor, Immunochemistry 8 (1971) 1081.

[2] Park et al., Colliodal Gold: Principles, Methods and Applications 1 (1989) M.A. Hayat, ed. Academic Press, Inc., 489.

[3] J. Slot and H. Geuze, Journal of Cell Biology 90 (1981) 533.

[4] G. Frens, Nature Physical Science 241 (1973) 20.

[5] D.A. Meyer and R.M. Albrecht, Microscopy and Microanalysis 8 (Suppl. 2) (2002)

[6] R.A. Zsigmondy, Zeitschrift für Physikalische Chemie 40 (1906) 697.

[7] F.P. Ottensmeyer, 1986. Ann. NY Acad. Sci. 483, 339.

[8] The authors gratefully acknowledge support from NIH/NIGMS grants \#63001 \& \#67244. 


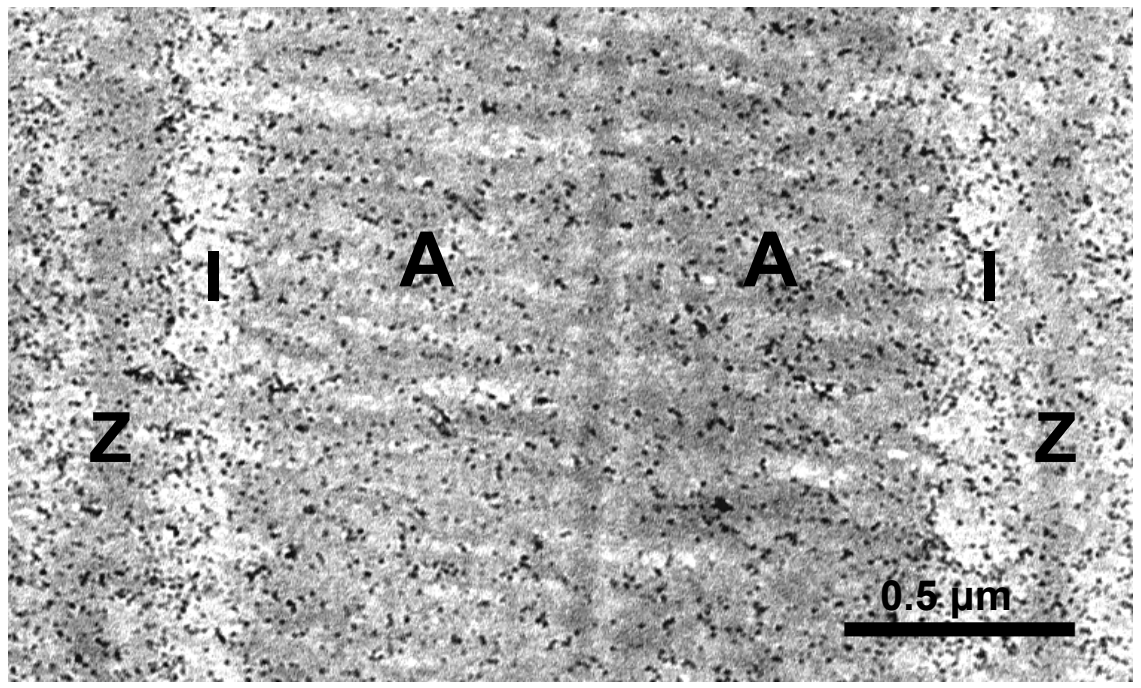

Fig. 1. Transmission electron micrograph of a multiple labeled cryosection of rat skeletal muscle tissue. Section was multiple labeled with anti-alpha-actinin-c $\mathrm{Au}_{6}$, (primarily on Z-bands), anti-actin-cPd (primarily on I-bands) and antimyosin-cPtAu ${ }_{6}$ conjugates (primarily on A-bands). $A=A$-band, $I=$ I-band, $\mathrm{Z}=\mathrm{Z}$-line.
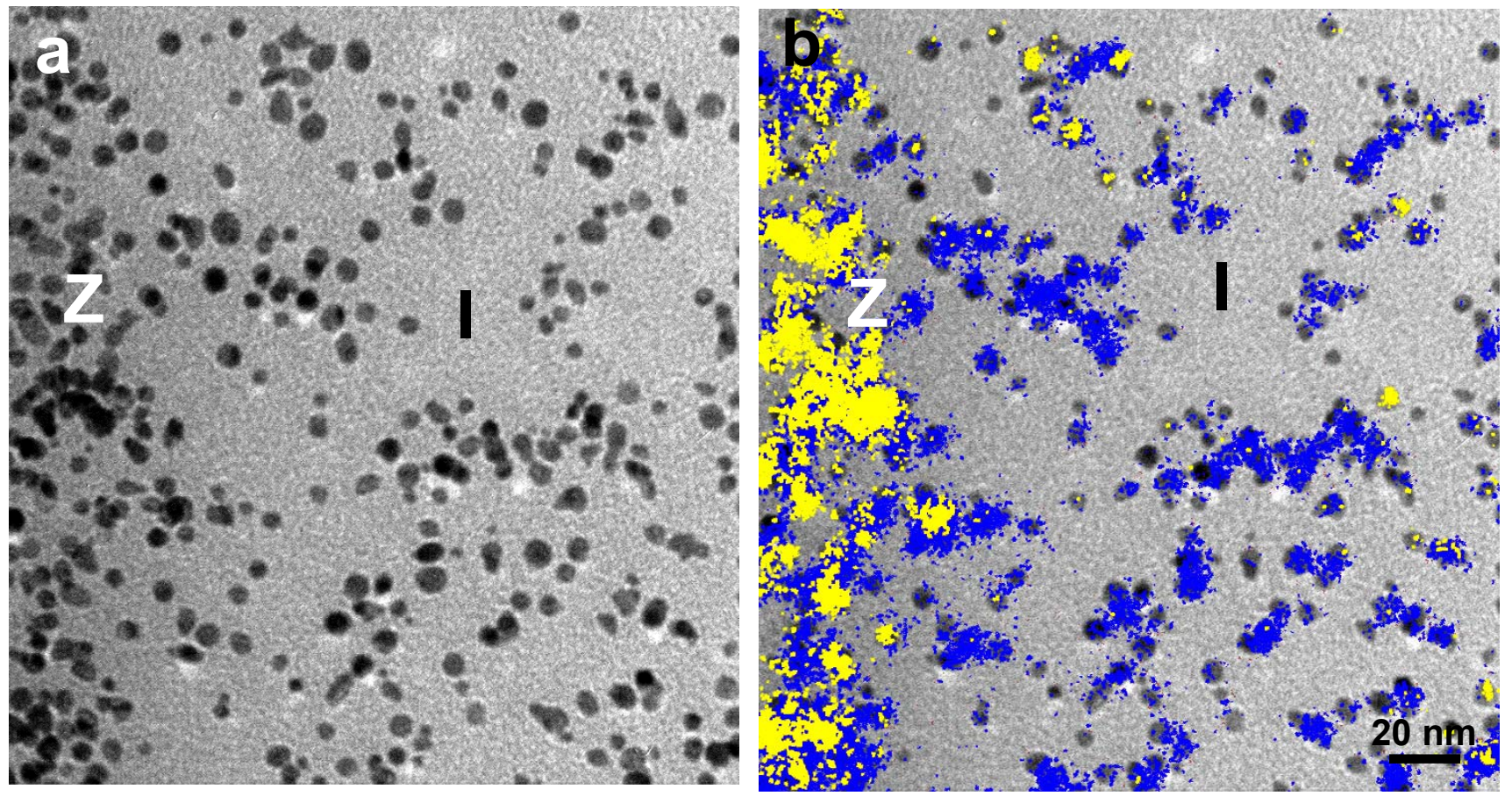

Fig. 2a. Zero-loss image over a Z-line $(Z)$ and adjacent I-band (I) of an ultrathin cryosection of rat skeletal muscle tissue labeled with anti $\alpha$-actinin-c $\mathrm{Au}_{6}$, anti-actin-cPd${ }_{6}$, and anti-myosin-cPtAu6 conjugates. Overlay of the elemental distribution of $\mathrm{Au}$ (yellow-light) and Pd (blue-dark) obtained by ESI with the three window method over the zero loss image is shown in b. Au specific signal, representing anti $\alpha$-actinin-cAu6 is found predominantly at the Z-line, and Pd-signal, corresponding to anti-actin-cPd $\mathrm{Cd}_{6}$ conjugates is present over the I-band and edges of Z-lines. A-band is not shown. 